\title{
Graviton production in brane worlds by the dynamical Casimir effect
}

\author{
Ruth Durrer, Marcus Ruser, Marc Vonlanthen and Peter Wittwer \\ Département de Physique Théorique, Université de Genève, 24, Quai E. Ansermet, 1211 Genève 4, \\ Switzerland
}

\begin{abstract}
If our Universe is a $3+1$ brane in a warped $4+1$ dimensional bulk so that its expansion can be understood as the motion of the brane in the bulk, the time dependence of the boundary conditions for arbitrary bulk fields can lead to particle creation via the dynamical Casimir effect. In this talk I report results for the simplest such scenario, when the only particle in the bulk is the graviton and the bulk is the 5 dimensional anti-de Sitter spacetime.
\end{abstract}

Keywords: Braneworlds, graviton production, dynamical Casimir effect

PACS: $98.80 \mathrm{Cq}, 04.50 .-\mathrm{h}, 04.30 .-\mathrm{W}$

\section{INTRODUCTION}

The idea that our Universe be a $3+1$ dimensional membrane in a higher dimensional 'bulk' spacetime has opened new exciting prospects for cosmology, for reviews see [1, 2]. In the simplest braneworlds motivated by string theory, the standard model particles are confined to the brane and only the graviton can propagate in the bulk. Of particular interest is the Randall-Sundrum (RS) model [3, 4], where the bulk is 5-dimensional anti-de Sitter space, $\mathrm{AdS}_{5}$. If the so called RS fine tuning condition is satisfied, it can be shown that gravity on the brane 'looks 4-dimensional' at low energies.

Within this model, cosmological evolution can be interpreted as the motion of the physical brane, i.e. our Universe, through the $5 \mathrm{~d}$ bulk. Such a time-dependent boundary does in general lead to particle production via the dynamical Casimir effect [5].

Of course one can always choose coordinates with respect to which the brane is at rest, e.g. Gaussian normal coordinates. But then usually (except in the case of de Sitter expansion on the brane [6]), the perturbation equation describing the evolution of gravitons is not separable and can be treated only with numerical simulations [7, 8, 9]. Furthermore, in a time-dependent bulk a mode decomposition is in general ambiguous and one cannot split the field in a zero mode and Kaluza-Klein (KK) modes in a unique way.

Based on the picture of a moving brane in $\mathrm{AdS}_{5}$, we have studied graviton production in an ekpyrotic type scenario [10] where our Universe first approaches a second static brane. After a 'collision' the physical brane reverses direction and moves away from the static brane, see Fig. 1. For an observer on the brane, the first phase corresponds to a contracting Universe and the collision represents the 'Big Bang' after which the Universe starts expanding.

Here I report on the results which we have obtained in our previous papers [11, 12, 13]. We have found that the energy density of KK gravitons in $\mathrm{AdS}_{5}$ scales like stiff matter, 


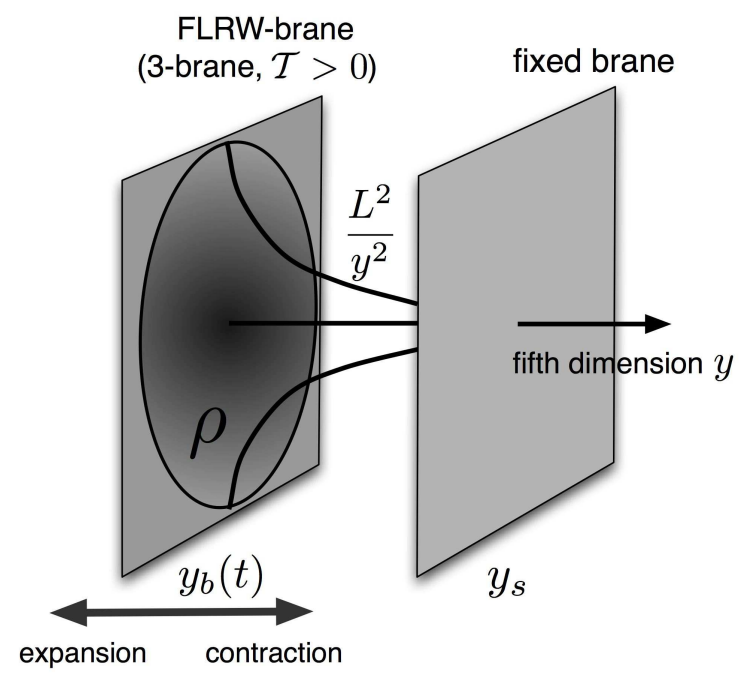

FIGURE 1. Two branes in an $\mathrm{AdS}_{5}$ spacetime. The physical brane is to the left. While it is approaching the static brane its scale factor is decreasing, the Universe is contracting, and when it moves away from the static brane the Universe is expanding. The AdS curvature radius $L$ (dashed line) and value of the scale factor of the brane metric as function of the extra dimension $y$ (light (blue) line) are also indicated.

$\rho_{\mathrm{KK}} \propto a^{-6}$, here $a$ denotes the scale factor defined in Eq. (2). Therefore, KK gravitons in $\mathrm{AdS}_{5}$ cannot represent the dark matter in the Universe. This finding is in contrast with the results of Ref. [14] and we comment on this below. We have also found that in the early Universe the back reaction from KK gravitons on the bulk geometry is likely to be important.

Finally, we have derived a limit for the maximal brane velocity, the bounce velocity, $v_{b} \lesssim 0.2$ in order not to over-produce zero-mode (i.e. $4 \mathrm{~d}$ ) gravitons, the energy density of which is constrained by the nucleosynthesis bound. We have calculated the spectra of both, the zero-mode and the KK gravitons. In Refs. [11, 12] we have, however, neglected a term linear in the brane velocity $v$ in the boundary conditions. In our latest work, Ref. [13] we derived a method which includes this term and allows to treat the problem without any low velocity approximation. We have shown that the low velocity results previously obtained are not modified.

The remainder of this paper is organized as follows. In the next section we present the basic equations for the evolution of tensor perturbations (gravitons) and we explain why it is not straight forward to include the velocity term of the boundary condition. In Section 3 we quantize the system. In Section 4 we discuss our results and in Section 5 we conclude. 


\section{A MOVING BRANE IN $\mathrm{AdS}_{5}$}

\subsection{The background}

In Poincaré coordinates $\left(x^{A}\right)=(t, \mathbf{x}, y)$ with $\mathbf{x}=\left(x^{1}, x^{2}, x^{3}\right)$ and $A=0, \ldots, 4$, the $\mathrm{AdS}_{5}$ (bulk) metric is given by

$$
d s^{2}=g_{A B} d x^{A} d x^{B}=\frac{L^{2}}{y^{2}}\left[-d t^{2}+\delta_{i j} d x^{i} d x^{j}+d y^{2}\right],
$$

where $i, j=1,2,3$ and $L$ is the $\mathrm{AdS}_{5}$ curvature radius which is related to the bulk cosmological constant by the $5 \mathrm{~d}$ Einstein equation, $-\Lambda=6 / L^{2}$. The physical brane representing our (spatially flat) Universe is located at some time dependent position $y=y_{b}(t)$ in the bulk, and the metric induced on the brane is the Friedman-RobertsonWalker metric,

$$
d s^{2}=a^{2}(\eta)\left[-\mathrm{d} \eta^{2}+\delta_{i j} d x^{i} d x^{j}\right]
$$

with scale factor $a(\eta)$ which is given by the brane position,

$$
a(\eta)=\frac{L}{y_{b}(t)}
$$

The conformal time $\eta$ of an observer on the brane, is related to the bulk time $t$ via

$$
d \eta=\sqrt{1-v^{2}} d t \equiv \gamma^{-1} d t
$$

Here we have introduced the brane velocity

$$
v \equiv \frac{d y_{b}}{d t}=-\frac{L H}{\sqrt{1+L^{2} H^{2}}} \quad \text { and } \gamma=\frac{1}{\sqrt{1-v^{2}}} .
$$

$H$ is the usual Hubble parameter,

$$
H \equiv \frac{1}{a^{2}} \frac{\partial a}{\partial \eta} \equiv a^{-1} \mathscr{H}=-L^{-1} \gamma \nu
$$

The brane dynamics, as a result of the second junction condition, is determined by the modified Friedmann equation [1]

$$
H^{2}=\frac{\kappa_{4} \rho}{3}\left(1+\frac{\rho}{2 \sigma}\right)
$$

where $\sigma$ is the brane tension, $\rho$ the energy density on the brane, and we assume the RS fine tuning condition [3]

$$
\frac{\kappa_{5}^{2} \sigma^{2}}{12}=\frac{3}{L^{2}}, \quad \text { and } \quad \kappa_{4} \equiv 8 \pi G_{4} \equiv \frac{\kappa_{5}^{2} \sigma}{6} .
$$


We define the string and Planck scales by

$$
\kappa_{5}=\frac{1}{M_{5}^{3}}=L_{s}^{3}, \quad \kappa_{4}=\frac{1}{M_{\mathrm{Pl}}^{2}}=L_{\mathrm{Pl}}^{2} .
$$

Note that the RS fine-tuning condition is equivalent to

$$
\kappa_{5}=\kappa_{4} L \text { or } \frac{L_{s}}{L}=\frac{L_{\mathrm{Pl}}^{2}}{L_{s}^{2}} .
$$

\subsection{Tensor perturbations}

We now consider $3 \mathrm{~d}$ tensor perturbations $h_{i j}(t, \mathbf{x}, y)$ of the spatial three-dimensional geometry on this background. The perturbed bulk metric reads

$$
d s^{2}=\frac{L^{2}}{y^{2}}\left[-d t^{2}+\left(\delta_{i j}+2 h_{i j}\right) d x^{i} d x^{j}+d y^{2}\right] .
$$

Tensor modes satisfy the traceless and transverse conditions, $h_{i}^{i}=\partial_{i} h_{j}^{i}=0$. These conditions imply that $h_{i j}$ has only two independent degrees of freedom, the two polarization states $\bullet=\times,+$. We decompose $h_{i j}$ into spatial Fourier modes,

$$
h_{i j}(t, \mathbf{x}, y)=\int \frac{d^{3} k}{(2 \pi)^{3 / 2}} \sum_{\bullet=+, \times} e^{i \mathbf{k} \cdot \mathbf{x}} e_{i j}^{\bullet}(\mathbf{k}) h_{\bullet}(t, y ; \mathbf{k}),
$$

where $e_{i j}^{\bullet}(\mathbf{k})$ are unitary constant transverse-traceless polarization tensors which form a basis of the two polarization states $\bullet=\times,+$. Since we assume parity symmetry, we shall neglect in the following the distinction between the two graviton polarizations and consider only one of them. We then have to multiply the final results for e.g. particle number or energy density by a factor of two to account for both polarizations.

The perturbed Einstein equations and the second junction condition lead to the following boundary value problem

$$
\left[\partial_{t}^{2}+k^{2}-\partial_{y}^{2}+\frac{3}{y} \partial_{y}\right] h(t, y ; \mathbf{k})=0 \text { in the bulk, } k^{2}=|\mathbf{k}|^{2},
$$

and

$$
\left.\gamma\left(v \partial_{t}+\partial_{y}\right) h\right|_{y_{b}(t)}=0 \text { on the brane . }
$$

We introduce also a second, static brane at position $y_{s}$, which requires the additional boundary condition

$$
\left.\partial_{y} h\right|_{y_{s}}=0 \text { on the static brane . }
$$

Eq. (13) is the Klein-Gordon equation for a minimally coupled massless mode in $\mathrm{AdS}_{5}$, i.e. the operator acting on $h$ is just the Klein-Gordon operator

$$
\square=\frac{1}{\sqrt{-g}} \partial_{A}\left[\sqrt{-g} g^{A B} \partial_{B}\right]
$$


Equation (14) is the time-dependent boundary condition (BC) coming from the fact that the moving brane acts like a "moving mirror" for the gravitational perturbations. Only in the rest-frame of the brane do we have pure Neumann BC. In a generic frame we have the Lorentz transformed $\mathrm{BC}$ which contains a velocity term $v \partial_{t}$.

We assume that the brane is filled with a perfect fluid such that there are no anisotropic stress perturbations in the brane energy momentum tensor, i.e. there is no coupling of gravitational waves to matter. If this were the case, the r.h.s. of Eq. (14) would not be zero but a term coupling $h_{i j}$ to the matter on the brane, see Eq. (2.25) of [12].

For the tensor perturbations the gravitational action up to second order in the perturbations reads

$$
\mathscr{S}_{h}=4 \frac{L^{3}}{2 \kappa_{5}} \int d t \int d^{3} k \int_{y_{b}(t)}^{y_{s}} \frac{d y}{y^{3}}\left[\left|\partial_{t} h\right|^{2}-\left|\partial_{y} h\right|^{2}-k^{2}|h|^{2}\right] .
$$

One factor of two in the action is due to $\mathbf{Z}_{2}$ symmetry while a second factor comes from the two polarizations.

\subsection{Dynamical Casimir effect approach}

The wave equation (13) itself has no time dependence and simply describes the propagation of free modes. It is the time dependence of the BC (14) that sources the non-trivial time-evolution of the perturbations. As it is well known, such a system of a wave equation and time-dependent $\mathrm{BC}$ lead, within a quantum mechanical formulation, to particle production from vacuum fluctuations. In the context of the photon field perturbed by a moving mirror this goes under the name "dynamical Casimir effect" [5].

In [12] we have extended a formalism which has been successfully employed for the numerical investigation of photon production in dynamical cavities [15, 16, 17] to the RS braneworld scenario. We have studied graviton production by a moving brane, which we call dynamical Casimir effect for gravitons, for a bouncing braneworld scenario.

However, in order to solve the problem, we have neglected the velocity term in Eq. (14). The ansatz

$$
h=\sum_{\alpha} a_{\alpha}(t) e^{-i \omega_{\alpha} t} \phi_{\alpha}(t, y)+\text { h.c. }, \omega_{\alpha}^{2}=k^{2}+m_{\alpha}(t)^{2}
$$

then leads to a Sturm-Liouville problem for the instantaneous eigenfunctions $\phi_{\alpha}(t, y)$ which satisfy

$$
\left(-\partial_{y}^{2}+\frac{3}{y} \partial_{y}\right) \phi_{\alpha}=m_{\alpha}^{2} \phi_{\alpha}
$$

The solutions of (18) are

$$
\begin{aligned}
\phi_{0}(t) & =\frac{y_{s} y_{b}(t)}{\sqrt{y_{s}^{2}-y_{b}^{2}(t)}}, \\
\phi_{n}(t, y) & =N_{n}(t) y^{2} C_{2}\left(m_{n}(t), y_{b}(t), y\right) \quad \text { with } \\
C_{v}(m, x, y) & =Y_{1}(m x) J_{v}(m y)-J_{1}(m x) Y_{v}(m y) .
\end{aligned}
$$


The function $\phi_{0}$ is the zero mode which corresponds to the ordinary $(3+1)$ d graviton on the brane while the $\phi_{n}$ are the KK modes. The masses $m_{n}$ are determined by the boundary condition at the static brane, see, e.g. [18] for more details. Since $\phi_{\alpha}$ satisfies Neumann boundary conditions, we know that the solutions $\left(\phi_{\alpha}\right)_{\alpha}$ form a complete orthonormal set of functions on the interval $\left[y_{b}(t), y_{s}\right]$ normalized by the scalar product

$$
\left(\phi_{\alpha}, \phi_{\beta}\right) \equiv 2 \int_{y_{b}(t)}^{y_{s}} \frac{d y}{y^{3}} \phi_{\alpha} \phi_{\beta}=\delta_{\alpha \beta}
$$

Therefore, any general solution which satisfies Neumann BC can be expanded in these instantaneous eigenfunctions. If we add the term $v \partial_{t}$ to the boundary condition this feature is lost, and we can no longer expect to find a complete set of instantaneous eigenfunctions.

However, since the entire effect disappears when the velocity tends to zero, neglecting a term which is first order in the velocity seems not to be consistent. This problem led us to search for another approach which is discussed in Ref. [13] where we transform to a coordinate system where the velocity term disappears identically. There also show that for low velocities $v<0.3$, say the corrections obtained with this consistent treatment are below a few percent. We therefore ignore it in the following.

\section{QUANTIZATION}

\subsection{Equation of motion}

The gravitational wave amplitude $h(t, y ; \mathbf{k})$ subject to Neumann boundary conditions can be expanded as

$$
h(t, y ; \mathbf{k})=\sqrt{\frac{\kappa_{5}}{L^{3}}} \sum_{\alpha=0}^{\infty} q_{\alpha, \mathbf{k}}(t) \phi_{\alpha}(t, y) .
$$

The coefficients $q_{\alpha, \mathbf{k}}(t)$ are canonical variables describing the time evolution of the perturbations and the factor $\sqrt{\kappa_{5} / L^{3}}$ has been introduced in order to render the $q_{\alpha, \mathbf{k}}$ 's canonically normalized. For $h(t, y, \mathbf{x})$ to be real, we have to impose the following reality condition on the canonical variables,

$$
q_{\alpha, \mathbf{k}}^{*}=q_{\alpha,-\mathbf{k}} .
$$

One could now insert the expansion (21) into the wave equation (13), multiply it by $\phi_{\beta}(t, y)$ and integrate out the $y$-dependence by using the orthonormality to derive the equations of motion for the variables $q_{\alpha, \mathbf{k}}$. However, as we explain in Refs. [12, 13], a Neumann boundary condition at a moving brane is not compatible with a free wave equation. The only consistent way to implement Neumann boundary conditions is therefore to consider the action (17) of the perturbations as the starting point to derive the equations of motion for $q_{\alpha, \mathbf{k}}$. Inserting (21) into (17) leads to the action

$$
\mathscr{S}=\frac{1}{2} \int d t \int d^{3} k\left\{\sum_{\alpha}\left[\left|\dot{q}_{\alpha, \mathbf{k}}\right|^{2}-\omega_{\alpha, k}^{2}\left|q_{\alpha, \mathbf{k}}\right|^{2}\right]+\right.
$$




$$
\left.\sum_{\alpha \beta}\left[M_{\alpha \beta}\left(q_{\alpha, \mathbf{k}} \dot{q}_{\beta,-\mathbf{k}}+q_{\alpha,-\mathbf{k}} \dot{q}_{\beta, \mathbf{k}}\right)+N_{\alpha \beta} q_{\alpha, \mathbf{k}} q_{\beta,-\mathbf{k}}\right]\right\} .
$$

We have introduced the time-dependent frequency of a graviton mode

$$
\omega_{\alpha, k}^{2}=\sqrt{k^{2}+m_{\alpha}^{2}}
$$

and the time-dependent coupling matrices

$$
\begin{aligned}
M_{\alpha \beta} & =\left(\partial_{t} \phi_{\alpha}, \phi_{\beta}\right), \\
N_{\alpha \beta} & =\left(\partial_{t} \phi_{\alpha}, \partial_{t} \phi_{\beta}\right)=\sum_{\gamma} M_{\alpha \gamma} M_{\beta \gamma}=\left(M M^{T}\right)_{\alpha \beta},
\end{aligned}
$$

which are given explicitely in Ref. [12] (see also [18]). The equations of motion for the canonical variables are the Euler-Lagrange equations from the action (23),

$$
\ddot{q}_{\alpha, \mathbf{k}}+\omega_{\alpha, k}^{2} q_{\alpha, \mathbf{k}}+\sum_{\beta}\left[M_{\beta \alpha}-M_{\alpha \beta}\right] \dot{q}_{\beta, \mathbf{k}}+\sum_{\beta}\left[\dot{M}_{\alpha \beta}-N_{\alpha \beta}\right] q_{\beta, \mathbf{k}}=0 .
$$

The motion of the brane through the bulk, i.e. the expansion of the universe, is encoded in the time-dependent coupling matrices $M_{\alpha \beta}$ and $N_{\alpha \beta}$. These mode couplings are caused by the time-dependent boundary condition $\left.\partial_{y} h_{\bullet}(t, y)\right|_{y_{b}}=0$ which forces the eigenfunctions $\phi_{\alpha}(t, y)$ to be explicitly time-dependent. In addition, the frequency of the KK modes $\omega_{\alpha, k}$ is also time-dependent since the distance between the two branes changes when the brane is in motion. Both time dependencies can lead to the amplification of tensor perturbations and, within a quantum treatment which is developed below, to graviton production from vacuum.

Because of translational invariance with respect to the directions parallel to the brane, modes with different $\mathbf{k}$ do not couple in (27). The three-momentum $\mathbf{k}$ enters the equation of motion for the perturbation only via the frequency $\omega_{\alpha, k}$. Equation (27) is similar to the equation describing the time evolution of electromagnetic field modes within a threedimensional dynamical cavity [16] and may effectively be described by a massive scalar field on a time-dependent interval [17]. For the electromagnetic field, the dynamics of the cavity, or more precisely the motion of one of its walls, leads to photon creation from vacuum fluctuations. This phenomenon is usually referred to as dynamical Casimir effect. Inspired by this, we call the production of gravitons by the moving brane the dynamical Casimir effect for gravitons.

\subsection{Quantization}

Asymptotically, i.e. for $t \rightarrow \pm \infty$, the physical brane approaches the Cauchy horizon $\left(y_{b} \rightarrow 0\right)$, moving very slowly. Then, the coupling matrices vanish and the KK masses become constant,

$$
\lim _{t \rightarrow \pm \infty} M_{\alpha \beta}(t)=0, \lim _{t \rightarrow \pm \infty} m_{\alpha}(t)=\text { const. } \forall \alpha, \beta .
$$


In this limit, the system (27) reduces to an infinite set of uncoupled harmonic oscillators. This allows to introduce an unambiguous and meaningful particle concept, i.e. the notion of (massive) gravitons.

Canonical quantization of the gravity wave amplitude is performed by replacing the canonical variables $q_{\alpha, \mathbf{k}}$ by the corresponding operators $\hat{q}_{\alpha, \mathbf{k}}$

$$
\hat{h}(t, y ; \mathbf{k})=\sqrt{\frac{\kappa_{5}}{L^{3}}} \sum_{\alpha} \hat{q}_{\alpha, \mathbf{k}}(t) \phi_{\alpha}(t, y) .
$$

Adopting the Heisenberg picture to describe the quantum time evolution, it follows that $\hat{q}_{\alpha, \mathbf{k}}$ satisfies the same equation (27) as the canonical variable $q_{\alpha, \mathbf{k}}$.

Under the assumptions outlined above, the operator $\hat{q}_{\alpha, \mathbf{k}}$ can be written for very early times, $t<t_{\text {in }}$, as

$$
\hat{q}_{\alpha, \mathbf{k}}\left(t<t_{\text {in }}\right)=\frac{1}{\sqrt{2 \omega_{\alpha, k}^{\text {in }}}}\left[\hat{a}_{\alpha, \mathbf{k}}^{\text {in }} e^{-i \omega_{\alpha, k}^{\text {in }} t}+\hat{a}_{\alpha,-\mathbf{k}}^{\mathrm{in} \dagger} e^{i \omega_{\alpha, k}^{\mathrm{in}} t}\right],
$$

where we have introduced the reference frequency

$$
\omega_{\alpha, k}^{\text {in }} \equiv \omega_{\alpha, k}\left(t<t_{\text {in }}\right) .
$$

This expansion ensures that Eq. (22) is satisfied. The set of annihilation and creation operators $\left\{\hat{a}_{\alpha, \mathbf{k}}^{\mathrm{in}}, \hat{a}_{\alpha, \mathbf{k}}^{\mathrm{in}}\right\}$ corresponding to the notion of gravitons for $t<t_{\text {in }}$ is subject to the usual commutation relations

$$
\begin{aligned}
& {\left[\hat{a}_{\alpha, \mathbf{k}}^{\text {in }}, \hat{a}_{\alpha^{\prime}, \mathbf{k}^{\prime}}^{\text {in } \dagger}\right]=\delta_{\alpha \alpha^{\prime}} \boldsymbol{\delta}^{(3)}\left(\mathbf{k}-\mathbf{k}^{\prime}\right),} \\
& {\left[\hat{a}_{\alpha, \mathbf{k}}^{\text {in }}, \hat{a}_{\alpha^{\prime}, \mathbf{k}^{\prime}}^{\text {in }}\right]=\left[\hat{a}_{\alpha, \mathbf{k}}^{\text {in }}, \hat{a}_{\alpha^{\prime}, \mathbf{k}^{\prime}}^{\text {in } \dagger}\right]=0 .}
\end{aligned}
$$

For very late times, $t>t_{\text {out }}$, i.e. after the motion of the brane has ceased, the operator $\hat{q}_{\alpha, \mathbf{k}}$ can be expanded in a similar manner,

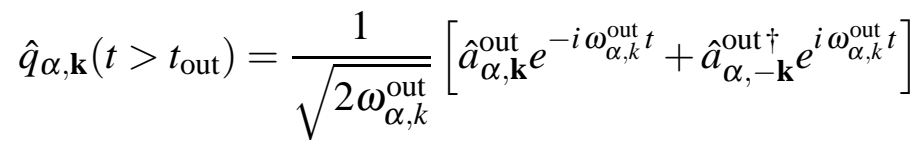

with final state frequency

$$
\omega_{\alpha, k}^{\text {out }} \equiv \omega_{\alpha, k}\left(t>t_{\text {out }}\right)
$$

The annihilation and creation operators $\left\{\hat{a}_{\alpha, \mathbf{k}}^{\text {out }}, \hat{a}_{\alpha, \mathbf{k}}^{\text {out }}\right\}$ correspond to a meaningful definition of final state gravitons (they are associated with positive and negative frequency solutions for $t \geq t_{\text {out }}$ ) and satisfy the same commutation relations as the initial state operators. 1 .

\footnotetext{
${ }^{1}$ Of course the brane never really stops moving, but before a certain time $t_{\text {in }}$ and after a certain time $t_{\text {out }}$ the motion is so slow that no particle production takes place. We have chosen these times sufficiently early (rsp. late) so that the numerical results are independent of their choice.
} 
Initial $\mid 0$,in $\rangle \equiv\left|0, t<t_{\text {in }}\right\rangle$ and final $\mid 0$, out $\rangle \equiv\left|0, t>t_{\text {out }}\right\rangle$ vacuum states are uniquely defined via 2

$$
\left.\left.\hat{a}_{\alpha, \mathbf{k}}^{\text {in }} \mid 0, \text { in }\right\rangle=0, \hat{a}_{\alpha, \mathbf{k}}^{\text {out }} \mid 0, \text { out }\right\rangle=0, \forall \alpha, \mathbf{k} .
$$

The operators counting the number of particles defined with respect to the initial and final vacuum state, respectively, are

$$
\hat{N}_{\alpha, \mathbf{k}}^{\text {in }}=\hat{a}_{\alpha, \mathbf{k}}^{\text {in } \dagger} \hat{a}_{\alpha, \mathbf{k}}^{\text {in }}, \hat{N}_{\alpha, \mathbf{k}}^{\text {out }}=\hat{a}_{\alpha, \mathbf{k}}^{\text {out } \dagger} \hat{a}_{\alpha, \mathbf{k}}^{\text {out }}
$$

The number of gravitons created during the motion of the brane for each momentum $\mathbf{k}$ and quantum number $\alpha$ is given by the expectation value of the number operator $\hat{N}_{\alpha, \mathbf{k}}^{\text {out }}$ of final-state gravitons with respect to the initial vacuum state $\mid 0$, in $\rangle$ :

$$
\mathscr{N}_{\alpha, \mathbf{k}}^{\text {out }}=\left\langle 0, \text { in }\left|\hat{N}_{\alpha, \mathbf{k}}^{\text {out }}\right| 0, \text { in }\right\rangle .
$$

If the brane undergoes a non-trivial dynamics between $t_{\text {in }}<t<t_{\text {out }}$ we have $\hat{a}_{\alpha, \mathbf{k}}^{\text {out }} \mid 0$, in $\rangle \neq$ 0 in general, i.e. graviton production from vacuum fluctuations takes place.

\section{RESULTS}

\subsection{Energy density}

For a usual four-dimensional tensor perturbation $h_{\mu \nu}$ on a background metric $g_{\mu \nu}$ an associated effective energy momentum tensor can be defined unambiguously by

$$
T_{\mu v}=\frac{1}{\kappa_{4}}\left\langle h_{\alpha \beta \| \mu} h_{\| v}^{\alpha \beta}\right\rangle
$$

where the bracket stands for averaging over several periods of the wave and " $\|$ " denotes the covariant derivative with respect to the unperturbed background metric. The energy density of gravity waves is the 00-component of the effective energy momentum tensor. We shall use the same effective energy momentum tensor to calculate the energy density corresponding to the four-dimensional spin-2 graviton component of the fivedimensional tensor perturbation on the brane, i.e. for the perturbation $h_{i j}\left(t, \mathbf{x}, y_{b}\right)$. For this it is important to remember that in our low energy approach, and in particular at very late times for which we want to calculate the energy density, the conformal time $\eta$ on the brane is identical to the conformal bulk time $t$. The energy density of fourdimensional spin-2 gravitons on the brane produced during the brane motion is then given by

$$
\rho=\frac{1}{\kappa_{4} a^{2}}\left\langle\left\langle 0, \text { in }\left|\dot{\hat{h}}_{i j}\left(t, \mathbf{x}, y_{b}\right) \dot{\hat{h}}^{i j}\left(t, \mathbf{x}, y_{b}\right)\right| 0, \text { in }\right\rangle\right\rangle .
$$

Here the outer bracket denotes averaging over several oscillations, which we embrace from the very beginning. The factor $1 / a^{2}$ comes from the fact that an over-dot indicates

\footnotetext{
2 Note that the notations $\left|0, t<t_{\text {in }}\right\rangle$ and $\left|0, t>t_{\text {out }}\right\rangle$ do not mean that the states are time-dependent; states do not evolve in the Heisenberg picture.
} 
the derivative with respect to conformal time $t \simeq \eta$. The detailed calculation given in Ref. [12] leads to

$$
\rho=\frac{2}{a^{4}} \sum_{\alpha} \int \frac{d^{3} k}{(2 \pi)^{3}} \omega_{\alpha, k} \mathscr{N}_{\alpha, k}(t) \mathscr{Y}_{\alpha}^{2}(a)
$$

where again $\mathscr{N}_{\alpha, k}(t)$ is the instantaneous particle number and $\mathscr{Y}_{\alpha}$ is related to value of the wave function on the brane by

$$
\mathscr{Y}_{\alpha}(a)=\frac{a}{L} \phi_{\alpha}\left(t, y_{b}(t)\right) \text {. }
$$

The factor two reflects the two polarizations. At late times, $t>t_{\text {out }}$, after particle creation has ceased, the energy density is

$$
\rho=\frac{2}{a^{4}} \sum_{\alpha} \int \frac{d^{3} k}{(2 \pi)^{3}} \omega_{\alpha, \mathrm{k}}^{\text {out }} \mathscr{N}_{\alpha, \mathbf{k}}^{\text {out }} \mathscr{Y}_{\alpha}^{2}(a) .
$$

This expression looks at first sight very similar to a "naive" definition of energy density as integration over momentum space and summation over all quantum numbers $\alpha$ of the energy $\omega_{\alpha, \mathbf{k}}^{\text {out }} \mathscr{N}_{\alpha, \mathbf{k}}^{\text {out }}$ of created gravitons. However, the important difference is the appearance of the function $\mathscr{Y}_{\alpha}^{2}(a)$ which exhibits a different dependence on the scale factor for the zero mode compared to the KK-modes.

Let us decompose the energy density into zero mode and $\mathrm{KK}$ contributions

$$
\rho=\rho_{0}+\rho_{K K}
$$

Evaluating $\mathscr{Y}_{0}(a)$ one then obtains for the energy density of the massless zero mode

$$
\rho_{0}=\frac{2}{a^{4}} \int \frac{d^{3} k}{(2 \pi)^{3}} k \mathscr{N}_{0, \mathbf{k}}^{\text {out }} .
$$

This is the expected behavior; the energy density of standard four-dimensional gravitons scales like radiation.

In contrast, the energy density of the KK-modes at late times is found to be

$$
\rho_{\mathrm{KK}}=\frac{L^{2}}{a^{6}} \frac{\pi^{2}}{2} \sum_{n=1}^{\infty} \int \frac{d^{3} k}{(2 \pi)^{3}} \omega_{n, k}^{\text {out }} \mathscr{N}_{n, \mathbf{k}}^{\text {out }} m_{n}^{2} Y_{1}^{2}\left(m_{n} y_{s}\right),
$$

which decays like $1 / a^{6}$. As the universe expands, the energy density of massive gravitons on the brane is therefore rapidly diluted. The total energy density of gravitational waves in our universe at late times is dominated by the standard four-dimensional graviton (massless zero mode). In the large mass limit, $m_{n} y_{s} \gg 1, n \gg 1$, the KK-energy density can be approximated by

$$
\rho_{\mathrm{KK}} \simeq \frac{\pi L^{2}}{2 a^{6} y_{s}} \sum_{n} \int \frac{d^{3} k}{(2 \pi)^{3}} \mathscr{N}_{n, \mathbf{k}}^{\text {out }} \omega_{n, k}^{\text {out }} m_{n} .
$$


Due to the factor $m_{n}$ coming from the function $\mathscr{Y}_{n}^{2}$, i.e. from the normalization of the functions $\phi_{n}(t, y)$, in order for the summation over the KK-tower to converge, the number of produced gravitons $\mathscr{N}_{n, \mathbf{k}}^{\text {out }}$ has to decrease faster than $1 / m_{n}^{3}$ for large masses and not just faster than $1 / m_{n}^{2}$ as one might naively expect.

\subsection{Escaping of massive gravitons and localization of gravity}

As we have shown, the energy density of the KK modes scales, at late times when particle production has ceased, with the expansion of the universe like

$$
\rho_{\mathrm{KK}} \propto 1 / a^{6}
$$

i.e. it decays by a factor $1 / a^{2}$ faster than the corresponding expression for the zero mode graviton and behaves effectively like stiff matter. Mathematically, this difference arises from the distinct behavior of the functions $\mathscr{Y}_{0}(a)$ and $\mathscr{Y}_{n}(a), n \geq 1$, and is a direct consequence of the warping of the fifth dimension which affects the normalization of the mode functions $\phi_{\alpha}$. But what is the underlying physics? As we shall discuss now, this scaling behavior for the KK particles has indeed a straight forward very appealing physical interpretation.

First, the mass $m_{n}$ is a comoving mass. The (instantaneous) 'comoving' frequency or energy of a KK graviton is $\omega_{n, k}=\sqrt{k^{2}+m_{n}^{2}}$, with comoving wave number $k$. The physical mass of a KK mode measured by an observer on the brane with cosmic time $d \tau=a d t$ is therefore $m_{n} / a$, i.e. the KK masses are redshifted with the expansion of the universe. This comes from the fact that $m_{n}$ is the wave number corresponding to the $y$ direction with respect to the bulk time $t$ which corresponds to conformal time $\eta$ on the brane and not to physical time. It implies that the energy of KK particles on a moving AdS brane redshifts like that of massless particles. From this alone one would expect the energy density of KK-modes on the brane to decay like $1 / a^{4}$ (see also Appendix D of [19]).

Now, let us define the normalized "wave function" for a graviton

$$
\Psi_{\alpha}(t, y)=\frac{\phi_{\alpha}(t, y)}{y^{3 / 2}}, \quad 2 \int_{y_{b}}^{y_{s}} d y \Psi_{\alpha}^{2}(t, y)=1 .
$$

From the expansion of the gravity wave amplitude Eq. (21) and the normalization condition it is clear that $\Psi_{n}^{2}(t, y)$ gives the probability to find a graviton of mass $m_{\alpha}$ for a given (fixed) time $t$ at position $y$ in the $\mathbf{Z}_{2}$-symmetric AdS-bulk.

In Fig. 2 we plot the evolution of $\Psi_{1}^{2}(t, y)$ under the influence of the brane motion with $v_{b}=0.1$. For this motion, the physical brane starting at $y_{b} \rightarrow 0$ for $t \rightarrow-\infty$ moves towards the static brane, corresponding to a contracting universe. After a bounce, it moves back to the Cauchy horizon, i.e. the universe expands. The second brane is placed at $y_{s}=10 L$ and $y$ ranges from $y_{b}(t)$ to $y_{s}$. As it is evident from this Figure, $\Psi_{1}^{2}$ is effectively localized close to the static brane, i.e. the weight of the KK-mode wave function lies in the region of less warping, far from the physical brane. Thus the probability to find a KK-mode is larger in the region with less warping. Since the effect 

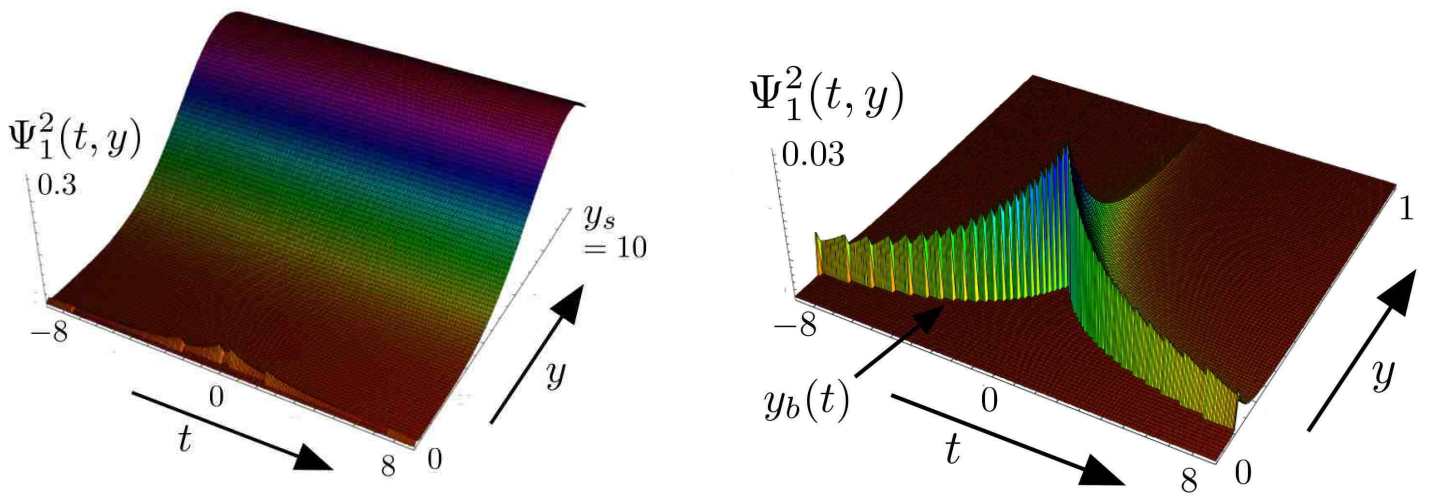

FIGURE 2. Evolution of $\Psi_{1}^{2}(t, y)=\phi_{1}^{2}(t, y) / y^{3}$ corresponding to the probability to find the first KK graviton at time $t$ at the position $y$ in the AdS-bulk. The static brane is at $y_{s}=10 \mathrm{~L}$ and the maximal brane velocity is given by $v_{b}=0.1$. On the right hand panel a zoom into the bulk-region close to the moving brane is shown.

of the brane motion on $\Psi_{1}^{2}$ is hardly visible in Fig. 2, we also show the behavior of $\Psi_{1}^{2}$ close to the physical brane (right hand panel).

This shows that $\Psi_{1}^{2}$ peaks also at the physical brane but with an amplitude roughly ten times smaller than the amplitude at the static brane. While the brane, coming from $t \rightarrow-\infty$, approaches the point of closest encounter, $\Psi_{1}^{2}$ slightly increases and peaks at the bounce $t=0$ where, as we shall see, the production of KK particles takes place. Afterwards, for $t \rightarrow \infty$, when the brane is moving back towards the Cauchy horizon, the amplitude $\Psi_{1}^{2}$ decreases again and so does the probability to find a KK particle at the position of the physical brane, i.e. in our universe. The parameter settings used in Fig. 2 are typical parameters which we use in the numerical simulations. However, the effect is illustrated much better if the second brane is closer to the moving brane. In Figure 3 (left panel) we show $\Psi_{1}^{2}$ for the same parameters as in Figure 2 but now with $y_{s}=L$. In this case, the probability to find a KK particle on the physical brane is of the same order as in the region close to the second brane during times close to the bounce. However, as the universe expands, $\Psi_{1}^{2}$ rapidly decreases at the position of the physical brane.

The behavior of the KK-mode wave function suggests the following interpretation: If KK gravitons are created on the brane, or equivalently in our universe, they escape from the brane into the bulk as the brane moves back to the Cauchy horizon, i.e. when the universe undergoes expansion. This is the reason why the power spectrum and the energy density imprinted by the KK-modes on the brane decrease faster with the expansion of the universe than for the massless zero mode.

The zero mode, on the other hand, is localized at the position of the moving brane. The profile of $\phi_{0}$ does not depend on the extra dimension, but the zero-mode wave function $\Psi_{0}$ does. Its square is

$$
\Psi_{0}^{2}(t, y)=\frac{y_{s}^{2} y_{b}^{2}}{y_{s}^{2}-y_{b}^{2}} \frac{1}{y^{3}} \rightarrow \frac{y_{b}^{2}}{y^{3}}=\left(\frac{L}{a}\right)^{2} \frac{1}{y^{3}} \text { if } y_{s} \gg y_{b}
$$


such that on the brane $\left(y=y_{b}\right)$ it behaves as

$$
\Psi_{0}^{2}\left(t, y_{b}\right) \simeq \frac{a}{L}
$$

Equation (49) shows that, at any time, the zero mode is localized at the position of the moving brane. For a better illustration we show Eq. (49) in Fig. 3, right panel for the same parameters as in the left panel. This is the "dynamical analog" of the localization mechanism for four-dimensional gravity discussed in [3, 4].
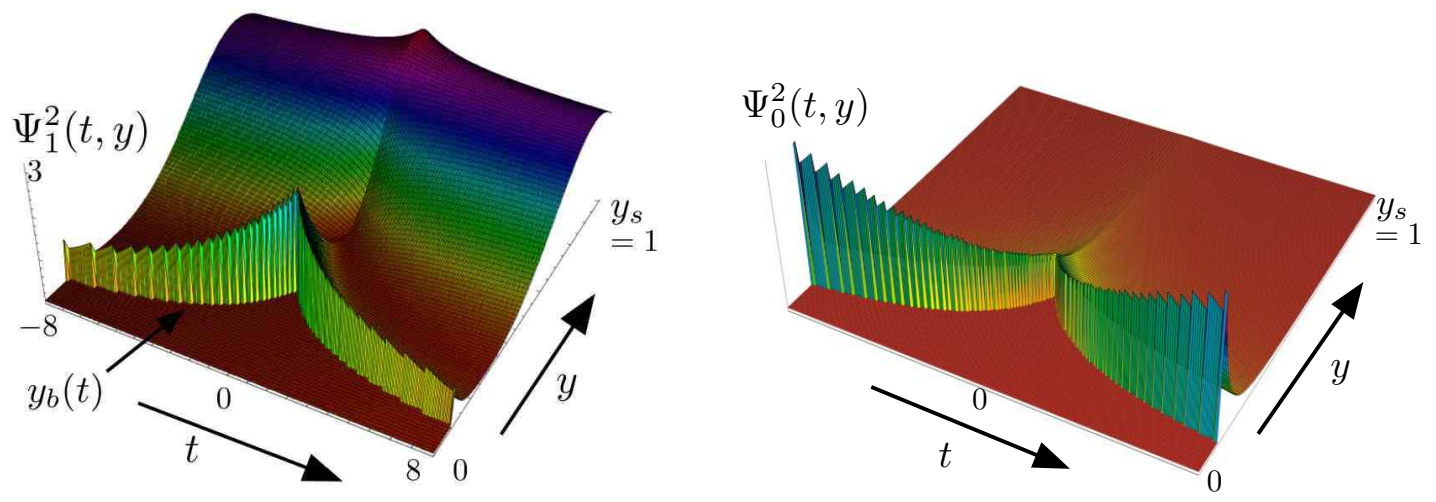

FIGURE 3. Left panel: evolution of $\Psi_{1}^{2}(t, y)$ for $y_{s}=L$ and $v_{b}=0.1$. Right panel: localization of fourdimensional gravity on a moving brane. Evolution of $\Psi_{0}^{2}(t, y)$. Note the opposite behavior of zero mode and massive mode.

This result is in contradiction with the findings of Ref. [14] where the authors conclude that for an observer on the brane KK gravitons behave like dust with a negative energy density. To arrive at this result, they use Gaussian normal coordinates,

$$
\begin{aligned}
d s^{2} & =-N^{2}(t, z) d t^{2}+Q^{2}(t, z) a^{2}(t) \delta_{i j} d x^{i} d x^{j}+d z^{2} \quad \text { with } \\
Q & =\cosh (z / L)-\gamma^{-1} \sinh (|z| / L) \quad N=\cosh (z / L)-\left(\gamma^{-1}-\frac{\dot{\gamma}}{\gamma^{2} H}\right) \sinh (|z| / L) \\
\gamma(t)^{-1} & =\sqrt{(H L)^{2}+1} \quad \text { see Eq. (5). }
\end{aligned}
$$

They then argue that at low velocity, $\gamma \simeq 1$, one may neglect the difference between $N$ and $Q$ so that one obtains the metric

$$
d s^{2} \simeq d z^{2}+e^{-2|z| / L}\left(-d t^{2}+a^{2}(t) \delta_{i j} d x^{i} d x^{j}\right) .
$$

In this metric, the mode equation for the KK modes separates and their time evolution can be determined by simply solving the time part of the equation, see [14]. There is, however, a flaw in this argument: the above approximation is only valid sufficiently close to the brane (which is positioned at $z \equiv 0$ in these coordinates), but far from the brane, when, e.g., $\left(\gamma^{-1}-1\right) \sinh (|z| / L)>\exp (-2|z| / L)$ the above metric is no longer a good approximation and the difference between $N$ and $Q$ does become important. As we have seen, the wave function of the KK gravitons actually is large far away from the brane and the time dependence enters in an important way in the normalization of the mode function which changes its scaling with time. 


\subsection{Spectra}

In Fig. 4 we show the results of a numerical simulation for three-momentum $k=$ $0.01 / L$, static brane position $y_{s}=10 L$ and maximal brane velocity $v_{b}=0.1$. Depicted is the graviton number for one polarization $\mathscr{N}_{\alpha, k}(t)$ for the zero mode and the first ten KKmodes as well as the evolution of the scale factor $a(t)$ and the position of the physical brane $y_{b}(t)$.

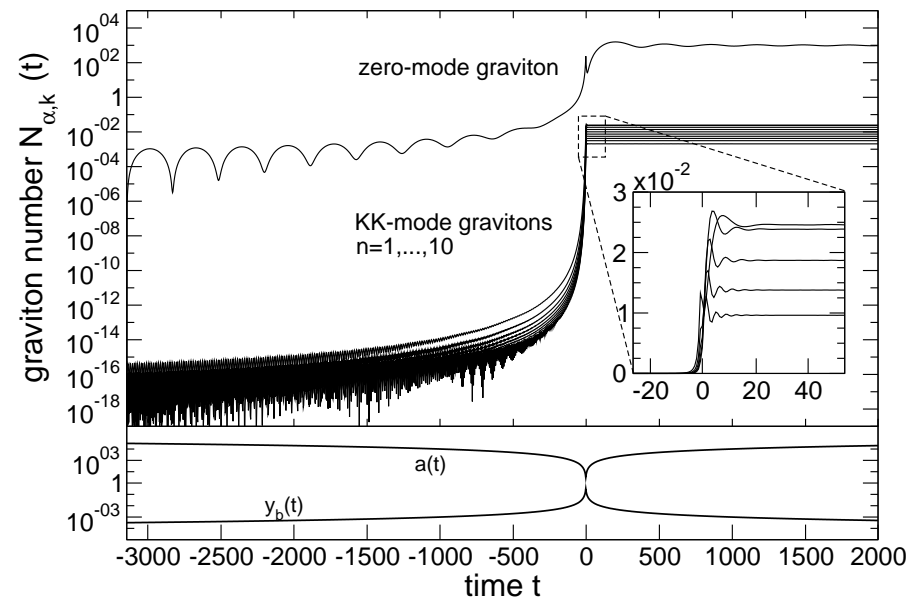

FIGURE 4. Evolution of the graviton number $\mathscr{N}_{\alpha, k}(t)$ for the zero mode (one polarization) and the first ten KK-modes for three-momentum $k=0.01 / L$ and $v_{b}=0.1, y_{s}=10 L$.

In Fig. [5 we show some KK spectra which we have obtained by integrating the equation of motion numerically. More details about the numerics and results for different values of the parameters can be found in Ref. [12]. In this paper we also derive an

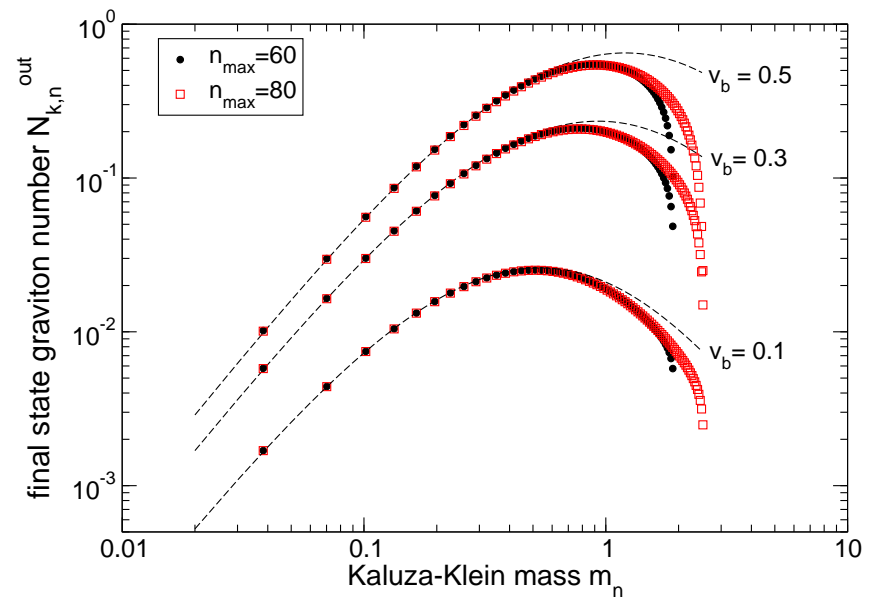

FIGURE 5. Final state KK graviton spectra for $k=0.001, y_{s}=100$, different maximal brane velocities $v_{b}$ at $t_{\mathrm{out}}=400$ for one polarization. The numerical results are compared with the analytical prediction (dashed line).

analytical approximation for the spectrum which is good for KK masses $m_{n}<1$. The numerical calculations are in very good agreement with the analytical estimates, where applicable. 
Integrating the zero-mode energy density over frequency with a cutoff given by the strong scale, $k_{\max }=1 / L_{s}$ leads to the following simple result for the gravitational wave density parameter [12]

$$
\Omega_{h 0} \simeq \frac{v_{b}}{2} \Omega_{\text {rad }} \quad \text { so that } \quad v_{b} \lesssim 0.2 .
$$

$\Omega_{\text {rad }}$ is the density parameter of the relativistic degrees of freedom at nucleosynthesis, the photon and three species of neutrini. The limit $v_{b}<0.2$ follows from the nucleosynthesis constraint which tells us that during nucleosynthesis $\Omega_{\text {rad }}$ should not deviate by more than $10 \%$ from its standard value [20]. The graviton spectrum is blue with tensor spectral index $n_{T}=2$. Its amplitude on Hubble scales is therefore severely suppressed and it leaves no detectable imprint on the cosmic microwave background [20].

Also the energy density of the KK modes grows like $k^{2}$ for suffiently large $k$,

$$
\frac{d \rho_{\mathrm{KK}}(k)}{d \log k} \propto k^{2}, \quad k \gtrsim 1
$$

and its maximum comes from the cutoff scale $k_{\max }=1 / L_{s}$. We find

$$
\rho_{\mathrm{KK}} \simeq \frac{\pi^{5} v_{b}^{2}}{a^{6} y_{s}} \frac{L^{2}}{L_{s}^{5}}, \quad\left(\frac{\rho_{\mathrm{KK}}}{\rho_{\mathrm{rad}}}\right)_{\max } \simeq 100 v_{b}^{3}\left(\frac{L}{y_{s}}\right)\left(\frac{L}{L_{s}}\right)^{2} .
$$

It is easy to see that low energy requires $y_{b}<L$ at all times. Therefore, to initiate a bounce, where $y_{b}$ should be close to $y_{s}$, we expect $y_{s} \lesssim L$. For typical values of the string scale, $L_{s} \ll L$ and $y_{s} \sim L$, the above ratio is not small and back reaction of the KK gravitons on the geometry has to be taken into account. The ratio indicated is the one directly after the big bang. As time goes on the KK mode energy density dilutes faster than radiation and rapidly becomes subdominant.

\section{CONCLUSIONS}

In braneworld cosmology where expansion is mimicked by a brane moving through a warped higher dimensional spacetime, the brane motion leads to particle creation via the dynamical Casimir effect for all bulk modes. Here we have studied the generation of gravitons.

The KK gravitons scale like stiff matter, $\rho_{\mathrm{KK}} \propto 1 / a^{6}$, and can therefore not represent dark matter. In an 'ekpyrotic type' scenario with an $\mathrm{AdS}_{5}$ bulk, the nucleosynthesis bound on gravitational waves requires $v_{b}<0.2$. Furthermore, back reaction of KK gravitons on the evolution of spacetime is most probably not negligible at early times.

In the RSII model where only one brane is present, graviton generation is negligible [18].

\section{ACKNOWLEDGMENTS}

RD thanks the Organizers of the Spanish Relativity meeting for inviting her to Salamanca, to assist and talk at this stimulating meeting. This work is supported by the 
Swiss National Science Foundation. Thanks go also the the Galileo Galilei Institut in Florence where part of the writing was done.

\section{REFERENCES}

1. R. Maartens, Living Rev. Rel. 7, 7 (2004). [e-Print: arXiv:gr-qc/0312059]

2. R. Durrer, Braneworlds, at the XI Brazilian School of Cosmology and Gravitation, Edt. M. Novello and S.E. Perez Bergliaffa, AIP Conference Proceedings 782 (2005). [e-Print: arXiv:hep-th/0507006]

3. L. Randall and R. Sundrum, Phys. Rev. Lett. 83, 3370 (1999). [e-Print: arXiv:hep-th/9905221]

4. L. Randall and R. Sundrum, Phys. Rev. Lett. 83, 4690 (1999). [e-Print: arXiv:hep-th/9906064]

5. M. Bordag, Quantum Field Theory under the Influence of External Conditions (Teuber, Stuttgart, 1996).

6. D. S. Gorbunov, V. A. Rubakov, and S. M. Sibiryakov, JHEP 10, 015 (2001). [e-Print: arXiv:hep-th/0108017]

7. T. Kobayashi, H. Kudoh, and T. Tanaka, Phys. Rev. D68, 044025 (2003); [e-Print: arXiv:gr-qc/0305006]

T. Kobayashi and T. Tanaka, Phys. Rev. D71, 124028 (2005);

[e-Print: arXiv:hep-th/0505065]

T. Kobayashi and T. Tanaka, Phys. Rev. D73, 044005 (2006).

[e-Print: arXiv:hep-th/0511186]

8. K. Koyama, JCAP 0409, 010 (2004).

[e-Print: arXiv:astro-ph/0407263]

9. S. Seahra, Phys. Rev. D74, 044010 (2006). [e-print: arXiv:hep-th/0602194v2]

10. J. Khoury, P. Steinhardt and N. Turok, Phys. Rev. Lett. 92, 031302 (2004), [e-print: arXiv:hep-th/0307132] Phys. Rev. Lett. 91161301 (2003). [e-print: arxivastro-ph/0302012]

11. R. Durrer and M. Ruser (2007), Phys. Rev. Lett. 99 , 071601. [e-Print:arXiv:0704.0756]

12. M. Ruser and Ruth Durrer, Phys. Rev. D 76, 104014 (2007). [e-Print:arXiv:0704.0790]

13. M. Ruser, R. Durrer, M. Vonlanthen and P. Wittwer, submitted (2009). [e-Print arXiv:0901.3683]

14. M. Minamitsuji, M. Sasaki and D. Langlois, Phys. Rev. D71, 084019 (2005). [e-print: arXiv:gr-qc/0501086]

15. M. Ruser, Opt. B: Quantum Semiclass. Opt. 7, S100 (2005). [e-print: arXiv:quant-ph/0408142]

16. M. Ruser, Phys. Rev. A73, 043811 (2006). [e-print: arXiv:quant-ph/0509030]

17. M. Ruser, J. Phys. A39 (2006) 6711. [e-print: arXiv:quant-ph/0603097]

18. C. Cartier, R. Durrer and M. Ruser, Phys. Rev. D72, 104018 (2005). [e-print: arXiv:hep-th/0510155]

19. D. S. Gorbunov, V. A. Rubakov and S. M. Sibiryakov, JHEP 10, 015 (2001). [e-print: arXiv:hep-th/0108017].

20. R. Durrer, The Cosmic Microwave Background, Cambridge University Press (2008). 